\title{
An editorial on the TAILOR trial-further support for the addition of cetuximab to FOLFOX-4 in the treatment of RAS wild-type metastatic colorectal cancer
}

\author{
Joanna Blanco, Michael Karass \\ Department of Internal Medicine, Westchester Medical Center, New York Medical College, Valhalla, NY, USA \\ Correspondence to: Michael Karass, MD. Department of Internal Medicine, Westchester Medical Center, New York Medical College, Valhalla, NY, \\ USA. Email: karass.michael@gmail.com. \\ Comment on: Qin S, Li J, Wang L, et al. Efficacy and Tolerability of First-Line Cetuximab Plus Leucovorin, Fluorouracil, and Oxaliplatin (FOLFOX-4) \\ Versus FOLFOX-4 in Patients With RAS Wild-Type Metastatic Colorectal Cancer: The Open-Label, Randomized, Phase III TAILOR Trial. J Clin \\ Oncol 2018:JCO2018783183. [Epub ahead of print].
}

Submitted Nov 27, 2018. Accepted for publication Nov 29, 2018.

doi: 10.21037/atm.2018.12.01

View this article at: http://dx.doi.org/10.21037/atm.2018.12.01

Worldwide, colorectal cancer (CRC) is the second most common cancer in women and the third most common cancer in men (1). In the United States, CRC continues to be a national burden with over 140,000 new cases of CRC reported in 2015 alone (2). Over the past two decades, there have been significant advances in CRC treatment resulting in an increase in the overall survival (OS) of patients diagnosed with unresectable metastatic CRC from 12 to 30 months (3).

In patients with $K R A S$ wild-type tumors, the addition of epidermal growth factor receptor (EGFR) inhibitor cetuximab to FOLFOX-4 (to fluorouracil, leucovorin, and oxaliplatin) remains controversial as there have been mixed results from previous trials. Overall, the United Kingdom MRC COIN trial and the NORDIC VII trial showed a lack of benefit in the addition of cetuximab to oxaliplatin-based chemotherapy $(4,5)$. The MRC COIN trial, which compared oxaliplatin and fluoropyrimidine chemotherapy (oral capecitabine $v$ intravenous fluorouracil and folinic acid) with and without cetuximab, showed an increase in overall response rate (ORR) but failed to show improvement in progression-free survival (PFS) time and OS (4). However, when comparing the different fluoropyrimidine regimens, there was improved PFS time in patients receiving fluorouracil-based therapy compared to capecitabine-based therapy [hazard ratio (HR) 0.77 for fluorouracil-based therapy vs. HR 1.06 for capecitabinebased therapy]. Capecitabine-based therapy was also noted to have more toxic effects, which resulted in a capecitabine dose reduction in the cetuximab arm. The NORDIC VII trial assigned patients to FLOX (fluorouracil, leucovorin, and oxaliplatin) alone, FLOX plus cetuximab, or cetuximab continuously and FLOX intermittently (5). ORR was higher, though nonsignificant, in the FLOX plus cetuximab arm (49\%) compared with FLOX alone (41\%), while there was no numerical or statistical difference in PFS or OS between the arms. It is unknown whether the bolus administration of fluorouracil in Nordic FLOX, when compared to the standard infusion in FOLFOX, impacts cetuximab therapy.

However, in the phase II OPUS trial, the addition of cetuximab to FOLFOX-4 illustrated a clinically significant higher response rate and lower risk of disease progression compared to treatment with FOLFOX-4 alone (6). In the TAILOR trial, Qin and colleagues sought to confirm the efficacy and safety of the addition of cetuximab to FOLFOX-4 in patients with $R A S$ wild-type metastatic CRC in a randomized phase III study of patients in China, published in the Fournal of Clinical Oncology (7). The primary end point was PFS time, while secondary end points included OS, ORR, and safety and tolerability.

A clinically significant increase in PFS time was seen in FOLFOX-4 plus cetuximab (9.2 months) versus FOLFOX-4 alone (7.4 months). This, however, is in the setting of a longer duration of oxaliplatin treatment for patients in the FOLFOX-4 plus cetuximab arm (25.8 vs. 
21 weeks with FOLFOX-4 plus cetuximab vs. FOLFOX-4 alone, respectively). Additionally, patients with FOLFOX-4 plus cetuximab had a statistically significant increase in OS (20.7 vs. 17.8 months with FOLFOX-4 plus cetuximab $v s$. FOLFOX-4 alone, respectively) and ORR (61.1\% vs. $39.5 \%$ with FOLFOX-4 plus cetuximab vs. FOLFOX-4 alone, respectively). Nearly every subgroup analysis favored the addition of cetuximab to FOLFOX-4 with the exception of patients with the $B R A F$ mutation, a mutational status that inhibits induction of immunogenic cell death and generally confers a poorer prognosis for patients (8). This supports a meta-analysis of the CRYSTAL and OPUS trials which comparably showed no significant improvement in PFS or OS for $B R A F$ mutant patients, a group that makes up less than $10 \%$ of CRC cases (8). Additionally, statistical significance for PFS time was demonstrated in patients less than 65 years of age but not in patients 65 years of age or older, a result likely secondary to the disproportionate number of participants in the younger age category (303 patients aged $<65 v s .90$ patients aged $\geq 65$ ). In a similar study, however, no difference was seen in PFS time between patients $<70$ years and those $\geq 70$ years, a valuable result since $>40 \%$ of CRC cases are diagnosed in patients aged 70 years or older (9).

The safety profile of FOLFOX-4 plus cetuximab was comparable to FOLFOX-4 alone with neutropenia being the most common grade $\geq 3$ adverse event (AE) in both arms. FOLFOX-4 plus cetuximab resulted in grade $\geq 3$ skin reactions for $25.8 \%$ of patients while there were no $\geq$ grade 3 skin reactions seen in FOLFOX-4 alone. However, as evidenced in the ObservEr trial, these skin reactions did not compromise the quality of life (QoL) of patients receiving cetuximab with chemotherapy (10). Furthermore, the addition of cetuximab to chemotherapy had comparable incidences of AEs and impact of QoL in patients grouped into those less than 70 years of age and those ages 70 years and older (9). In the FOLFOX-4 plus cetuximab arm, $19.1 \%$ of patients experienced serious $\mathrm{AE}$ resulting in $39.2 \%$ of patients in that group discontinuing chemotherapy, while in the FOLFOX-4 arm alone, 13.1\% of patients had serious $\mathrm{AE}$ resulting in $27.1 \%$ discontinuing therapy.

Given the clinically significant increase in PFS, OS, and ORR and the acceptable safety profile of the addition of cetuximab to chemotherapy seen in the phase III TAILOR trial, Qin and colleagues help support the use of FOLFOX-4 plus cetuximab as first-line therapy for treatment of $R A S$ wild-type metastatic CRC without $B R A F$ mutation status in patients of all ages. Promising research looking into new predictive biomarkers for cetuximab therapy will hopefully further direct strategies for treating $R A S$ wild-type metastatic CRC (11).

\section{Acknowledgements}

None.

\section{Footnote}

Conflicts of Interest: The authors have no conflicts of interest to declare.

\section{References}

1. Ferlay J, Soerjomataram I, Dikshit R, et al. Cancer incidence and mortality worldwide: sources, methods and major patterns in GLOBOCAN 2012. Int J Cancer 2015;136:E359-86.

2. U.S. Cancer Statistics Working Group. U.S. Cancer Statistics Data Visualizations Tool, based on November 2017 submission data (1999-2015): U.S. Department of Health and Human Services, Centers for Disease Control and Prevention and National Cancer Institute; www.cdc. gov/cancer/dataviz, June 2018.

3. Fakih MG. Metastatic colorectal cancer: current state and future directions. J Clin Oncol 2015;33:1809-24.

4. Maughan TS, Adams RA, Smith CG, et al. Addition of cetuximab to oxaliplatin-based first-line combination chemotherapy for treatment of advanced colorectal cancer: results of the randomised phase 3 MRC COIN trial. Lancet 2011;377:2103-14.

5. Tveit KM, Guren T, Glimelius B, et al. Phase III trial of cetuximab with continuous or intermittent fluorouracil, leucovorin, and oxaliplatin (Nordic FLOX) versus FLOX alone in first-line treatment of metastatic colorectal cancer: the NORDIC-VII study. J Clin Oncol 2012;30:1755-62.

6. Bokemeyer C, Bondarenko I, Hartmann JT, et al. Efficacy according to biomarker status of cetuximab plus FOLFOX-4 as first-line treatment for metastatic colorectal cancer: the OPUS study. Ann Oncol 2011;22:1535-46.

7. Qin S, Li J, Wang L, et al. Efficacy and Tolerability of First-Line Cetuximab Plus Leucovorin, Fluorouracil, and Oxaliplatin (FOLFOX-4) Versus FOLFOX-4 in Patients With RAS Wild-Type Metastatic Colorectal Cancer: The Open-Label, Randomized, Phase III TAILOR Trial. J Clin Oncol. 2018:JCO2018783183. [Epub ahead of print]. 
8. Pozzi C, Cuomo A, Spadoni I, et al. The EGFR-specific antibody cetuximab combined with chemotherapy triggers immunogenic cell death. Nat Med 2016;22:624-31.

9. Rosati G, Pinto C, Di Fabio F, et al. Quality of life, compliance, safety and effectiveness in fit older metastatic colorectal patients with cancer treated in first-line with chemotherapy plus cetuximab: A restrospective analysis from the ObservEr study. J Geriatr Oncol 2018;9:243-8.

10. Pinto C, Di Fabio F, Rosati G, et al. Observational

Cite this article as: Blanco J, Karass M. An editorial on the TAILOR trial-further support for the addition of cetuximab to FOLFOX-4 in the treatment of RAS wild-type metastatic colorectal cancer. Ann Transl Med 2018;6(Suppl 2):S114. doi: 10.21037/atm.2018.12.01 study on quality of life, safety, and effectiveness of firstline cetuximab plus chemotherapy in KRAS wild-type metastatic colorectal cancer patients: the ObservEr Study. Cancer Med 2016;5:3272-81.

11. Zheng P, Liang C, Ren L, et al. Additional Biomarkers beyond RAS That Impact the Efficacy of Cetuximab plus Chemotherapy in mCRC: A Retrospective Biomarker Analysis. J Oncol 2018 Sep 16;2018:5072987. 